\title{
Síndrome autoinmune inflamatorio inducido por adyuvantes (ASIA): síndrome de Shoenfeld
}

\author{
Autoimmune/inflammatory syndrome induced by \\ adjuvants (ASIA): Shoenfeld's syndrome
}

\author{
Luis Gerardo Domínguez Carrillo, * José Gregorio Arellano Aguilar \\ Citar como: Domínguez CLG, Arellano AJG. Síndrome autoinmune inflamatorio inducido por adyuvantes (ASIA): \\ síndrome de Shoenfeld. Acta Med GA. 2022; 20 (1): 96-98. https://dx.doi.org/10.35366/103566
}

\section{Resumen}

Introducción: El síndrome autoinmune inflamatorio inducido por adyuvantes es una entidad descrita en 2011. Sus manifestaciones incluyen mialgias, artralgias, fatiga crónica y boca seca, así como manifestaciones neurológicas: alteraciones cognitivas, pérdida de memoria y discapacidades neurológicas. Caso clínico: Mujer de 40 años, con prótesis mamarias de 20 años, que en los últimos seis meses ha presentado: mialgias, fatiga, sensación de rigidez articular y artralgias generalizadas, incluyendo columna cervical y lumbar, dificultad para concentrase en su trabajo y alteraciones del sueño, cambios en estado anímico con irritabilidad y disnea al subir escaleras, incrementándose la fatiga principalmente en extremidades pélvicas. Los estudios de laboratorio relevantes mostraron insuficiencia de vitamina $\mathrm{D}$, elevación de proteína $\mathrm{C}$ reactiva y velocidad de sedimentación globular (VSG) incrementada; la mamografía reportó contracturas tipo II de Baker. Se efectuó retiro de prótesis mamarias y prescripción de vitamina $D$, a seis meses del postoperatorio, paciente asintomática efectuando ejercicio aeróbico cinco días de la semana. Conclusión: Los implantes de silicón pueden llegar a producir el síndrome de ASIA en pacientes inmunológicamente susceptibles.

Palabras clave: Síndrome autoinmune por adyuvantes, prótesis mamaria, silicón.

\section{Abstract}

Introduction: Adjuvant-induced inflammatory autoimmune syndrome is an entity described in 2011. Its manifestations include myalgias, arthralgias, chronic fatigue and dry mouth, as well as neurological manifestations: cognitive alterations, memory loss and neurological disabilities. Clinical case: 40-year-old female, with breast prostheses for 20 years, who in the last six months has presented: myalgias, fatigue, sensation of joint stiffness and generalized arthralgias, including cervical and lumbar spine, difficulty concentrating at work and sleep disturbances, mood changes with irritability and dyspnea when climbing stairs, increasing fatigue mainly in the pelvic extremities. Relevant laboratory studies showed vitamin $D$ insufficiency, elevated C-reactive protein and increased erythrocyte sedimentation rate (ESR); mammography reported Baker's type II contractures. Breast prosthesis was removed and vitamin D was prescribed, six months postoperatively, the patient was asymptomatic and underwent aerobic exercise five days a week. Conclusion: Silicone implants can produce ASIA syndrome in immunologically susceptible patients.

Keywords: Adjuvant autoimmune syndrome, breast prosthesis, silicone.

un conjunto de enfermedades mediadas por mecanismos inmunitarios, que comparten un cuadro clínico común, así como el antecedente de exposición previa a un agente adyuvante (una sustancia capaz de aumentar la inmunogenicidad de un antígeno sin despertar una respuesta per

El síndrome autoinmune inflamatorio inducido por adyuvantes (ASIA, por sus siglas en inglés) es una entidad descrita por Shoenfeld ${ }^{1}$ y colaboradores en 2011. Constituye

* Catedrático de la Facultad de Medicina de León, Universidad de Guanajuato. México.

₹ Médico Internista. División de Medicina del Hospital Angeles León. León, Guanajuato, México.

\section{Correspondencia: \\ Dr. Luis Gerardo Domínguez Carrillo \\ Correo electrónico: Igdominguez@hotmail.com}

Aceptado: 14-04-2021.

www.medigraphic.com/actamedica 
se). Este fenómeno inmunitario se ha reportado en cuatro entidades como son: 1) síndrome de miofascitis macrofágica (MMF); 2) fenómeno postvacunación; 3) síndrome de la guerra del Golfo (SGG); y 4) siliconosis. ${ }^{2}$ Se cree que los fenómenos posteriores a la vacunación y el MMF se desarrollan principalmente tras el uso de adyuvantes a base de aluminio, mientras que la incidencia del SGG se debe a la exposición al adyuvante de escualeno. Desde el punto de vista clínico, los pacientes con ASIA presentan mialgias, artralgias, fatiga crónica y boca seca, así como manifestaciones neurológicas como alteraciones cognitivas, pérdida de memoria y discapacidades neurológicas. La etiopatogenia de estas condiciones implica una interacción multifactorial entre factores ambientales y predisposición genética, existiendo asociación con ciertos haplotipos del complejo mayor de histocompatibilidad. Desde su aparición como entidad patológica, se han informado más de 4,000 casos documentados de síndrome ASIA con diversa gravedad clínica y antecedentes diversos de exposición al adyuvante. Dado que en el mundo los implantes de mama son muy frecuentes, es importante recordar que éstos no son inocuos, pudiendo causar problemas, que pueden pasar desapercibidos o confundir al clínico. Al presentarse una paciente con la sintomatología antes mencionada, se efectúa la presentación del caso, considerando que puede ser importante para el médico general, el ginecoobstetra, el reumatólogo y el cirujano plástico tener en mente al síndrome de ASIA.

\section{CASO CLÍNICO}

Mujer de 40 años, con antecedente de implante de prótesis mamarias 20 años atrás. Sin antecedentes de importancia. Acude a consulta por presentar un cuadro clínico de seis meses de evolución, caracterizado por mialgias generalizadas, fatiga, sensación de rigidez articular y artralgias generalizadas, incluyendo columna cervical y lumbar, principalmente matutinas con duración de 30 a 40 minutos, dificultad para concentrase en su trabajo y alteraciones del sueño, despertando muy frecuentemente. En los últimos dos meses se agregaron cambios en estado anímico con irritabilidad y disnea al subir escaleras, incrementándose la fatiga principalmente en extremidades pélvicas. A la exploración, los datos a llamar la atención fueron: frecuencia cardiaca (FC) 85 lat./min; frecuencia respiratoria (FR) 18 resp./min; tensión arterial (TA) 110/60 mmHg; peso $62 \mathrm{~kg}$, talla $167 \mathrm{~cm}$; índice de masa corporal (IMC) 22.2 . Cuello con ingurgitación venosa notoria (Figura 1), tiroides no palpable, arcos de movimiento cervical normales con presencia de dolor moderado al efectuarlos, contractura de paravertebrales cervicales, trapecio superior y angular del omóplato bilateral, ausencia de puntos gatillo, presencia de ganglios axilares incrementados en tamaño moderadamente dolorosos. Columna lumbar con aumento de tono muscular en paravertebrales lumbares, con arcos de movilidad normales moderadamente dolorosos a la flexoextensión, y ausencia de puntos gatillo; extremidades torácicas con examen clínico muscular con calificación de 5/5 en escala de Daniels, pero con sensación de fatiga contra contracción resistida. Reflejos osteotendinosos, sensibilidad y llenado capilar normales.

Se consideraron las siguientes posibilidades diagnósticas: 1) fibromialgia, 2) hipotiroidismo, 3) artritis reumatoide, 4) síndrome de ASIA (por prótesis mamarias) y 5) cardiopatía a descartar. Dentro de los resultados de laboratorio destacaron: hidroxivitamina $\mathrm{D}$ en $12.66 \mathrm{ng} / \mathrm{mL}$; proteína C reactiva $15 \mathrm{mg} / \mathrm{dL}$; velocidad de sedimentación globular en $49 \mathrm{~mm}$, resto dentro de los parámetros normales. La radiografía de tórax y ecocardiografía normales. La mamografía mostró contracturas tipo II de Baker (Figura 2), y el ultrasonido mamario, reportó ganglios incrementados de volumen a nivel axilar.

Se llegó al diagnóstico de síndrome de ASIA por presentar al menos cuatro criterios mayores (mialgias, artralgias, fatiga y alteraciones del sueño). Se explicó a la paciente el diagnóstico, así como la relación existente entre el silicón de las prótesis y su sintomatología, por lo que se determinó el retiro de las prótesis por el Departamento de Cirugía Plástica, así como la administración de vitamina D. La evolución fue favorable, presentándose asintomática a los seis meses postcirugía, realizando ejercicio aeróbico cinco días a la semana, con control de hidroxivitamina D dentro de parámetros normales.

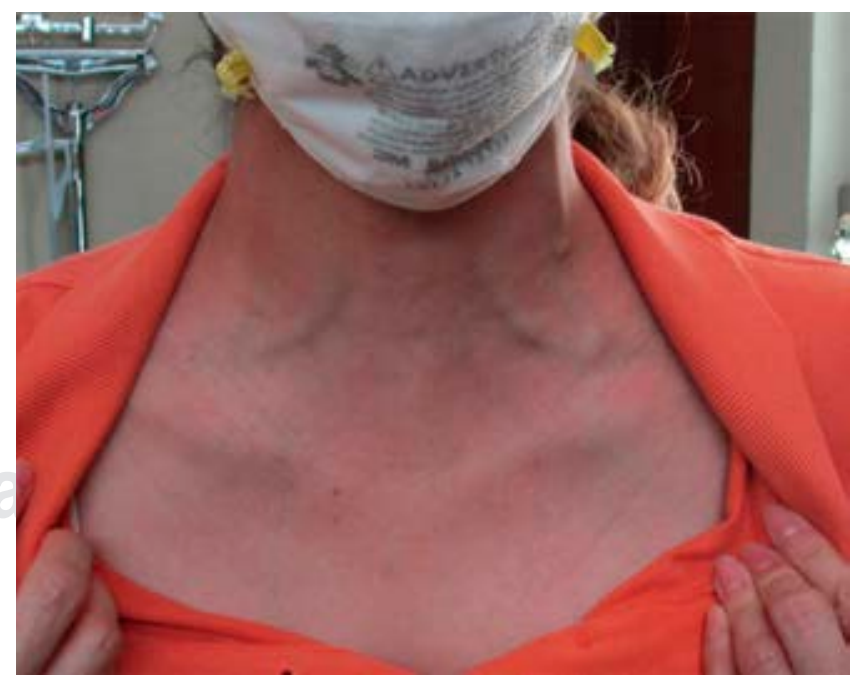

Figura 1: Fotografía clínica de mujer de 40 años con ingurgitación venosa notoria durante el reposo y sin maniobras de Valsalva. 

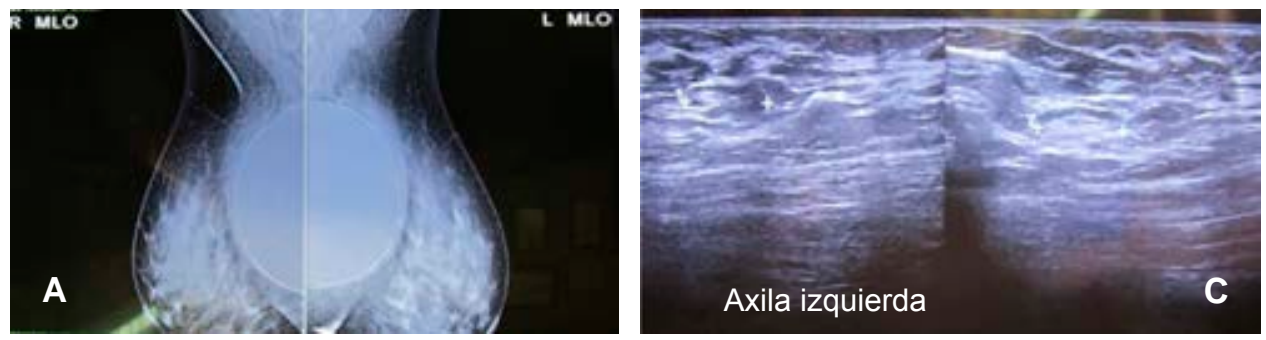

Figura 2:
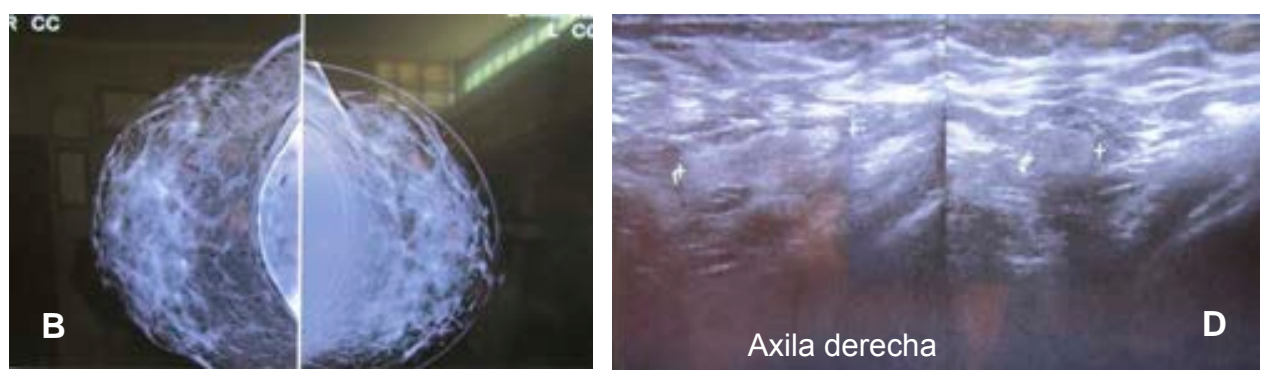

A, B) Imágenes de mamografía bilateral mostrando ambas mamas catalogadas como BlRADS-2, y presencia de implantes mamarios, existiendo contracturas tipo II de Baker. C, D) Imágenes de ultrasonido a nivel axilar, mostrando incremento bilateral de tamaño de dos ganglios axilares.

\section{DISCUSIÓN}

Los adyuvantes son moléculas que funcionan potenciando las respuestas inmunitarias específicas del antígeno, en sí mismos no generan una respuesta inmune, auxilian en la producción de una reacción importante contra sus antígenos inoculados. Por lo general, los adyuvantes influyen tanto en la inmunidad adaptativa como innata, activando los receptores de reconocimiento de patrones, específicamente en los receptores de tipo Toll (TLR), los receptores de tipo NOD y los receptores de lectina de tipo C, conduciendo al reclutamiento y activación de proteínas y generación de citocinas; promueven la quimiotaxis de las células dendríticas y la activación de las células presentadoras de antígenos, incrementando la transferencia de antígenos a las áreas con abundantes células B y T. ${ }^{2}$ En individuos genéticamente susceptibles la exposición de adyuvantes puede inducir síntomas musculoesqueléticos constitucionales generalizados inespecíficos, y puede conducir a la producción de autoanticuerpos y la inducción de enfermedades autoinmunes. ${ }^{3}$

El reporte de Fryzek ${ }^{4}$ y colaboradores mostró resultados estadísticamente significativos (RR con variación de 1.2 a 1.4, con intervalo de confianza de $95 \%$ ) en relación con los síntomas constitucionales y síntomas reumatológicos en 1,546 pacientes con implante de silicón, en comparación con 2,496 controles; estos hallazgos coinciden en la posible relación entre el implante de silicón y la agrupación de estos síntomas, constituyendo al síndrome ASIA. Otro factor involucrado es el nivel bajo de vitamina $D$ relacionado con el desarrollo de autoinmunidad, incluyendo desarrollo de anticuerpos, situación que se ve incrementada si se asocia con presencia de silicón, ${ }^{5}$ lo que sugiere el posible beneficio de la acción inmunomoduladora de la vitamina $\mathrm{D}$ en la prevención de la formación posterior de anticuerpos en estas pacientes.

\section{CONCLUSIONES}

Las pacientes con síndrome de ASIA con implantes de silicón que poseen ciertos haplotipos genéticos, incluidos HLA-DR5 y HLA-DQ2, tienen mayor probabilidad y predisposición de presentar el cuadro, por lo que se justifica retirar los implantes de silicón cuando las pacientes presenten este tipo de manifestaciones clínicas.

\section{REFERENCIAS}

1. Shoenfeld Y, Agmon-Levin N. 'ASIA'- autoimmune/inflammatory syndrome induced by adjuvants. J Autoimmun. 2011; 36 (1): 4-8.

2. Watad A, Sharif K, Shoenfeld Y. The ASIA syndrome: basic concepts. Mediterr J Rheumatol. 2017; 28 (2): 64-69. doi: 10.31138/ mjr.28.2.64.

3. Watad A, Bragazzi NL, Amital H, Shoenfeld Y. Hyperstimulation of adaptive immunity as the common pathway for silicone breast implants, autoimmunity, and lymphoma of the breast. Isr Med Assoc J. 2019; 21 (8): 517-519.

Fryzek JP, Signorello LB, Hakelius L, Feltelius N, Ringberg A, Blot WJ et al. Self-reported symptoms among women after cosmetic breast implant and breast reduction surgery. Plast Reconstr Surg. 2001; 107 (1): 206-213.

5. Colaris MIL, van der Hulst RR, Tervaert JWC. Vitamin D deficiency as a risk factor for the development of autoantibodies in patients with ASIA and silicone breast implants: a cohort study and review of the literature. Clin Rheumatol. 2017; 36 (5): 981-993. 